\title{
Representing Topological Relationships between Complex Regions by F-Histograms
}

\author{
Lukasz Wawrzyniak, Pascal Matsakis, Dennis Nikitenko \\ Department of Computing and Information Science \\ University of Guelph, Guelph, ON, N1G 2W1, Canada \\ \{lwawrzyn, matsakis, dnikiten\}@ cis.uoguelph.ca
}

\begin{abstract}
In earlier work, we introduced the notion of the F-histogram and demonstrated that it can be of great use in understanding the spatial organization of regions in images. Moreover, we have recently designed $F$ histograms coupled with mutually exclusive and collectively exhaustive relations between line segments. These histograms constitute a valuable tool for extracting topological relationship information from $2 D$ concave objects. For any direction in the plane, they define a fuzzy partition of all object pairs, and each class of the partition corresponds to one of the above relations. The present paper continues this line of research. It lays the foundation for generating a linguistic description that captures the essence of the topological relationships between two regions in terms of the thirteen Allen relations. An index to measure the complexity of the relationships in an arbitrary direction is developed, and experiments are performed on real data.
\end{abstract}

\section{Introduction}

Work in the modeling of topological relationships often relies on an extension into the spatial domain of Allen's temporal relations (Allen 1983). Although several alternatives and refinements have been proposed, a common procedure is to approximate the geometry of spatial objects by Minimum Bounding Rectangles (Nabil et al. 1995; Sharma and Flewelling 1995). Many authors, e.g., (Goodchild and Gopal 1990), have stressed the need to handle imprecise and uncertain information about spatial data. Qualitative spatial reasoning aims at modeling commonsense knowledge of space. Nevertheless, computational approaches for spatial modeling and reasoning can benefit from more quantitative measures, and the interest of fuzzy approaches has been widely recognized (Dutta 1991; Freeman 1975).

In previous publications, we introduced the notion of the F-histogram (Matsakis 1998; Matsakis and Wendling 1999), a generic quantitative 
representation of the relative position between two 2D objects. Most work focused on particular F-histograms called force histograms. As demonstrated in (Matsakis 2002), these histograms can be of great use in understanding the spatial organization of regions in images. For instance, they can provide inputs to systems for linguistic scene description (Matsakis et al. 2001). Moreover, we have recently shown (Matsakis and Nikitenko, to appear) that the F-histogram constitutes a valuable tool for extracting topological relationship information from 2D concave objects. The present paper builds both on (Matsakis et al. 2001) and (Matsakis and Nikitenko, to appear). It lays the foundation for generating a linguistic description that captures the essence of the topological relationships between two complex regions in terms of the thirteen Allen relations. The notion of the F-histogram is briefly described in Sect. 2. The way Fhistograms can be coupled with Allen relations using fuzzy set theory is examined in Sect. 3. Section 4 describes experiments on real data. It shows that the F-histograms associated with a given pair of objects carry lots of topological relationship information. An index to measure the complexity of the relationships in an arbitrary direction is developed in Sect. 5. This index will play an important role in the generation of linguistic descriptions. Conclusions are given in Sect. 6.

\section{F-Histograms}

As shown in Fig. 1, the plane reference frame is a positively oriented orthonormal frame $(\mathrm{O}, \vec{i}, \vec{j})$. For any real numbers $\alpha$ and $\mathrm{v}$, the vectors $\vec{i}_{\alpha}$ and $\vec{j}_{\alpha}$ are the respective images of $\vec{i}$ and $\vec{j}$ through the $\alpha$-angle rotation, and $\Delta_{\alpha}(\mathrm{v})$ is the oriented line whose reference frame is defined by $\vec{i}_{\alpha}$ and the point of coordinates $(0, \mathrm{v})$ - relative to $\left(\mathrm{O}, \vec{i}_{\alpha}, \overrightarrow{ }_{\alpha}\right)$. An object is a nonempty bounded set of points, E, equal to its interior closure ${ }^{1}$, and such that for any $\alpha$ and $\mathrm{v}$ the intersection $\mathrm{E}_{\alpha}(\mathrm{v})=\mathrm{E} \cap \Delta_{\alpha}(\mathrm{v})$ is the union of a finite number of mutually disjoint segments. An object may have holes in it and may consist of many connected components. $\mathrm{E}_{\alpha}(\mathrm{v})$ is a longitudinal section of E. Finally, $T$ denotes the set of all triples $\left(\alpha, \mathrm{E}_{\alpha}(\mathrm{v}), \mathrm{G}_{\alpha}(\mathrm{v})\right)$, where $\alpha$ and $v$ are any real numbers and $E$ and $G$ are any objects.

Now, consider two objects A and B (the argument and the referent), a direction $\theta$ and some proposition $\mathcal{P}^{\mathrm{AB}}(\theta)$ like "A is after $\mathrm{B}$ in direction $\theta$," "A overlaps B in direction $\theta$," or "A surrounds B in direction $\theta$." We want to attach a weight to $\mathscr{P}^{\mathrm{AB}}(\theta)$. To do so, the objects $\mathrm{A}$ and $\mathrm{B}$ are handled as longitudinal sections.

1 In other words, it is a 2D object that does not include any "grafting," such as an arc or isolated point. 
- For each $\mathrm{v}$, the pair $\left(\mathrm{A}_{\theta}(\mathrm{v}), \mathrm{B}_{\theta}(\mathrm{v})\right)$ of longitudinal sections is viewed as an argument put forward to support $\mathcal{P}^{\mathrm{AB}}(\theta)$.

- A function $\mathrm{F}$ from $\mathrm{T}$ into $\mathbb{R}_{+}$(the set of non-negative real numbers) attaches the weight $\mathrm{F}\left(\theta, \mathrm{A}_{\theta}(\mathrm{v}), \mathrm{B}_{\theta}(\mathrm{v})\right)$ to this argument $\left(\mathrm{A}_{\theta}(\mathrm{v}), \mathrm{B}_{\theta}(\mathrm{v})\right)$.

- The total weight $\mathrm{F}^{\mathrm{AB}}(\theta)$ of the arguments stated in favor of $\mathcal{P}^{\mathrm{AB}}(\theta)$ is naturally set to (Fig. 2): $\mathrm{F}^{\mathrm{AB}}(\theta)=\int_{-\infty}^{+\infty} \mathrm{F}\left(\theta, \mathrm{A}_{\theta}(\mathrm{v}), \mathrm{B}_{\theta}(\mathrm{v})\right) \mathrm{dv}$.

The function $\mathrm{F}^{\mathrm{AB}}$ so defined is called the $\mathrm{F}$-histogram associated with $(\mathrm{A}, \mathrm{B})$. It is one possible representation of the position of A with regard to B. F-histograms include f-histograms, which include $\varphi$-histograms, which themselves include force histograms (Matsakis 1998; Matsakis and Nikitenko, to appear). Most work has focused on force histograms (Matsakis 2002). Malki et al. (2002), however, use f-histograms ${ }^{2}$ to attach weights to the propositions $\mathcal{P}_{r}^{\mathrm{AB}}(\theta) \equiv$ "A $r \mathrm{~B}$ in direction $\theta$," where $r$ belongs to the set $\{>, m i, o i, f, d, s i,=, s, d i, f i, o, m,<\}$ of Allen relations (Fig. 3). But the thirteen f-histograms are not defined in a consistent manner and only convex objects are considered. The work of Malki et al. is discussed and revisited in a book chapter by Matsakis and Nikitenko (to appear). The F-histograms designed in that chapter are presented in Sect. 3.

\section{F-Histograms and Allen Relations}

Consider a set of mutually exclusive and collectively exhaustive relations between segments of an oriented line. F-histograms can be coupled with such relations using fuzzy set theory. We consider here the well-known set of Allen relations (Fig. 3). More details about the F-histograms described below can be found in (Matsakis and Nikitenko, to appear).

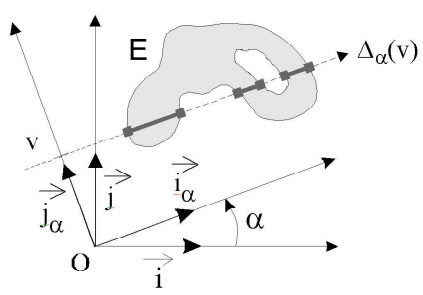

Fig. 1. Oriented straight lines and longitudinal sections. $\mathrm{E}_{\alpha}(\mathrm{v})=\mathrm{E} \cap \Delta_{\alpha}(\mathrm{v})$ is here the union of three segments.

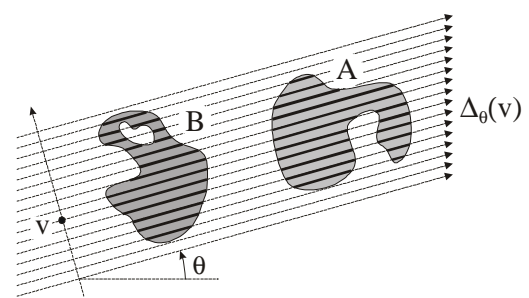

Fig. 2. The objects $A$ and $B$ are handled as longitudinal sections: $\mathrm{F}^{\mathrm{AB}}(\theta)=\int_{-\infty}^{+\infty} \mathrm{F}\left(\theta, \mathrm{A} \cap \Delta_{\theta}(\mathrm{v}), \mathrm{B} \cap \Delta_{\theta}(\mathrm{v})\right) \mathrm{dv}$.

2 Although the authors rely on the research presented in (Matsakis 1998), they refer to these f-histograms as the histogram of spatial relations. In their publications, they also use the term of orientation histogram instead of $\varphi$-histogram or force histogram. We do not subscribe to these changes in terminology. 


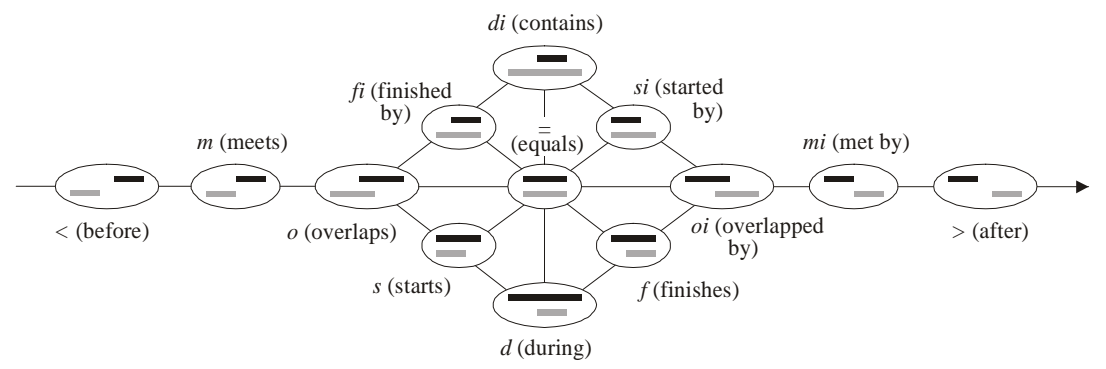

Fig. 3. Allen relations (Allen 1983) between two segments of an oriented line. The black segment is the referent, the gray segment is the argument. Two relations $r_{1}$ and $r_{2}$ are linked if and only if they are conceptual neighbors, i.e., $r_{l}$ can be obtained directly from $r_{2}$ by moving or deforming the segments in a continuous way.

Let $r$ denote an Allen relation, A and B two objects (convex or not), and $\theta$ a direction. To attach a weight to the proposition $\mathcal{P}_{r}^{\mathrm{AB}}(\theta) \equiv$ "A $r \mathrm{~B}$ in direction $\theta$," each pair $\left(\mathrm{A}_{\theta}(\mathrm{v}), \mathrm{B}_{\theta}(\mathrm{v})\right)$ of longitudinal sections is viewed as an argument put forward to support $\mathcal{P}_{r}^{\mathrm{AB}}(\theta)$ (Sect. 2). A function $\mathrm{F}_{r}$ attaches the weight $\mathrm{F}_{r}\left(\theta, \mathrm{A}_{\theta}(\mathrm{v}), \mathrm{B}_{\theta}(\mathrm{v})\right)$ to this argument, and the total weight $\mathrm{F}_{r}^{\mathrm{AB}}(\theta)$ of the arguments stated in favor of $\mathcal{P}_{r}^{\mathrm{AB}}(\theta)$ is set to:

$$
\mathrm{F}_{r}^{\mathrm{AB}}(\theta)=\int_{-\infty}^{+\infty} \mathrm{F}_{r}\left(\theta, \mathrm{A}_{\theta}(\mathrm{v}), \mathrm{B}_{\theta}(\mathrm{v})\right) \mathrm{dv}
$$

The question, of course, is how to define $\mathrm{F}_{r}$. Small changes in the longitudinal sections should not affect $\mathrm{F}_{r}^{\mathrm{AB}}(\theta)$ significantly. Fuzzy set theoretic approaches have been widely used to handle imprecision and achieve robustness in spatial analysis. Allen relations are fuzzified in Sect. 3.1 and longitudinal sections in Sect. 3.2. The last section, Sect. 3.3, defines the function $\mathrm{F}_{r}$.

\subsection{Fuzzification of Allen Relations}

An Allen relation $r$ can be fuzzified in many ways, depending on the intent of the work. For instance, Guesgen (2002) proceeds in a qualitative manner. Here, we proceed in a quantitative manner. The 13 Allen relations are fuzzified as shown in Fig. 4. Each relation, except $=$, is defined by the min of a few trapezoid membership functions. Let $\mathcal{A}$ be the set of all thirteen fuzzy relations. Three properties are worth noticing. First, for any pair (I,J) of segments, we have $\Sigma_{r \in \mathcal{A}} r(\mathrm{I}, \mathrm{J})=1$, where $r(\mathrm{I}, \mathrm{J})$ denotes the degree to which the statement $\mathrm{I} r \mathrm{~J}$ is to be considered true. This, of course, comes from the definition of $=$ (and it can be shown that $=$ takes its values in $[0,1])$. Second, for any $r$ in $\mathcal{A}$, there exist pairs $(\mathrm{I}, \mathrm{J})$ such that $r(\mathrm{I}, \mathrm{J})=1$. Lastly, for any pair (I,J) and any $r_{1}$ and $r_{2}$ in $\mathcal{A}$, if $r_{I}(\mathrm{I}, \mathrm{J}) \neq 0$ and $r_{2}(\mathrm{I}, \mathrm{J}) \neq 0$ then $r_{1}$ and $r_{2}$ are direct neighbors in the graph of Fig. 3 . 


\subsection{Fuzzification of Longitudinal Sections}

The idea is to consider that if two segments are close enough relative to their lengths, then they should be seen, to a certain extent, as a single segment. Let I be the longitudinal section $\mathrm{E}_{\theta}(\mathrm{v})$ of some object E. Assume I is not empty. There exists one set $\left\{I_{i}\right\}_{i \in 1 . . n}$ (and only one) of mutually disjoint segments such that: $I=\cup_{i \in 1 . . n} I_{i}$. The indexing can be chosen such that, for any $\mathrm{i}$ in $1 . . \mathrm{n}-1$, the segment $I_{i+1}$ is after $I_{i}$ in direction $\theta$. Let $\mathrm{J}_{\mathrm{i}}$ be the open interval "between" $\mathrm{I}_{\mathrm{i}}$ and $I_{i+1}$. The longitudinal section $\mathrm{I}$ is considered a fuzzy set on $\Delta_{\theta}(\mathrm{v})$ with membership function $\mu_{\mathrm{I}}$. For any point $\mathrm{M}$ on any $\mathrm{I}_{\mathrm{i}}$, the value $\mu_{\mathrm{I}}(\mathrm{M})$ is 1 . For any point $\mathrm{M}$ on any $\mathrm{J}_{\mathrm{i}}$, the value $\mu_{\mathrm{I}}(\mathrm{M})$ is $\alpha_{i}$-and, initially, $\alpha_{i}=0$. Fuzzification of I proceeds by increasing these membership degrees $\alpha_{i}$. An example is presented in Fig. 5. Details can be found in (Matsakis and Nikitenko, to appear).

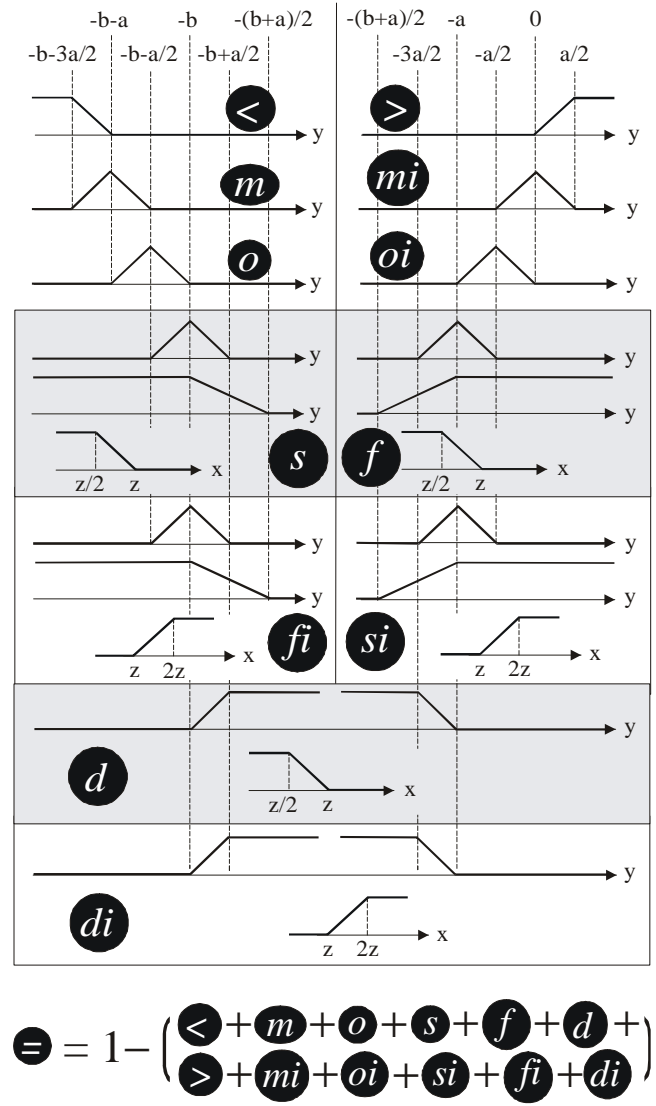

Fig. 4. Fuzzified Allen relations between two segments I and $\mathrm{J}$ of an oriented line. Each relation, except $=$, is defined by the min of a few membership functions (one for $\langle,>, m, m i, o, o i$; three for $s, s i, f, f i$; and two for $d$ and $d i$ ). $\mathrm{x}$ is the length of I (the argument), $\mathrm{z}$ is the length of $\mathrm{J}$ (the referent $), a=\min (x, z), b=\max (x, z)$ and $y$ is the signed distance from the end of $\mathrm{J}$ to the start of $\mathrm{I}$.

\subsection{Coupling F-Histograms with Allen Relations}

Consider an Allen relation $r$ and the longitudinal sections $\mathrm{A}_{\theta}(\mathrm{v})$ and $\mathrm{B}_{\theta}(\mathrm{v})$ of some objects $\mathrm{A}$ and $\mathrm{B}$. We are now able to define the value $\mathrm{F}_{r}\left(\theta, \mathrm{A}_{\theta}(\mathrm{v}), \mathrm{B}_{\theta}(\mathrm{v})\right)$ 
(a)

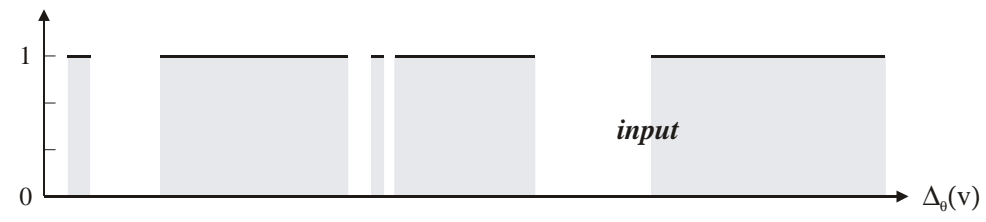

(b)

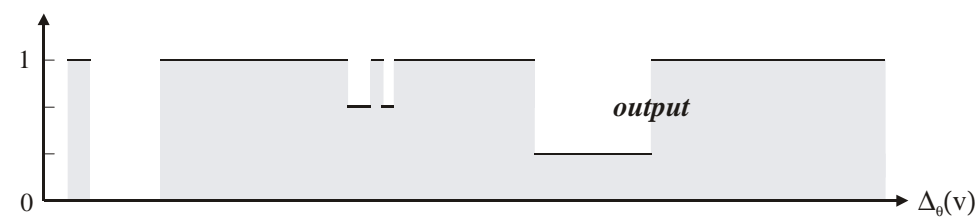

Fig. 5. Fuzzification of a longitudinal section I. (a) Membership function $\mu_{\mathrm{I}}$ before fuzzification. (b) Membership function after fuzzification.

(see the introductory paragraph of Sect. 3). If $A_{\theta}(v)=\varnothing$ or $B_{\theta}(v)=\varnothing$ then $\mathrm{F}_{r}\left(\theta, \mathrm{A}_{\theta}(\mathrm{v}), \mathrm{B}_{\theta}(\mathrm{v})\right)$ is naturally set to 0 . Assume $\mathrm{A}_{\theta}(\mathrm{v}) \neq \varnothing$ and $\mathrm{B}_{\theta}(\mathrm{v}) \neq \varnothing$. Assume $r, \mathrm{~A}_{\theta}(\mathrm{v})$ and $\mathrm{B}_{\theta}(\mathrm{v})$ have been fuzzified as described in Sects. 3.1 and 3.2. There exists a tuple $\left(\alpha_{0}, \alpha_{1}, \ldots, \alpha_{c}\right)$ of real numbers such that $\alpha_{0}=0<\alpha_{1}<\alpha_{2}<\ldots<\alpha_{c}=1$ and $\left\{\alpha_{k}\right\}_{\mathrm{k} \in 0 . . .}=\left\{\mu_{\mathrm{A}_{\theta}(\mathrm{v})}(\mathrm{M})\right\}_{\mathrm{M} \in \Delta_{\theta}(\mathrm{v})} \cup\left\{\mu_{\mathrm{B} \theta(\mathrm{v})}(\mathrm{M})\right\}_{\mathrm{M} \in \Delta_{\theta}(\mathrm{v})}$ (the set of all membership values in the fuzzy sections $A_{\theta}(v)$ and $B_{\theta}(v)$ ). For any $\mathrm{k}$ in 1..c, there exists one set $\left\{\mathrm{I}_{\mathrm{i}}^{\mathrm{k}}\right\}_{\mathrm{i} \in 1 . . \mathrm{m}_{\mathrm{k}}}$ of mutually disjoint segments such that the $\alpha_{k}$-cut $\alpha_{k} A_{\theta}(v)$ is equal to $\cup_{i \in 1 . . m_{k}} I_{i}^{k}$. Likewise, there exists one set $\left\{J_{j}^{k}\right\}_{j \in 1 . . n_{k}}$ of segments such that $\alpha_{k} B_{\theta}(v)=\cup_{j \in 1 . . n_{k}} J_{j}^{k}$. For any $i$ in $1 . . m_{k}$, the length of $I_{i}^{k}$ is denoted by $x_{i}^{k}$. For any $j$ in $1 . . n_{k}$, the length of $J_{j}^{k}$ is denoted by $z_{j}^{k}$. The value $F_{r}\left(\theta, A_{\theta}(v), B_{\theta}(v)\right)$ is defined as ${ }^{3}$ :

$\mathrm{F}_{r}\left(\theta, \mathrm{A}_{\theta}(\mathrm{v}), \mathrm{B}_{\theta}(\mathrm{v})\right)=\frac{\mathrm{x}+\mathrm{z}}{\mathrm{w}} \sum_{\mathrm{k} \in 1 . . \mathrm{c}} \Sigma_{\mathrm{i} \in 1 . . \mathrm{m}_{\mathrm{k}}} \Sigma_{\mathrm{j} \in 1 . . \mathrm{n}_{\mathrm{k}}}\left[\mathrm{x}_{\mathrm{i}}^{\mathrm{k}} \mathrm{z}_{\mathrm{j}}^{\mathrm{k}}\left(\alpha_{\mathrm{k}}-\alpha_{\mathrm{k}-1}\right)\right] r\left(\mathrm{I}_{\mathrm{i}}^{\mathrm{k}}, \mathrm{J}_{\mathrm{j}}^{\mathrm{k}}\right)$,

with $\mathrm{x}=\Sigma_{\mathrm{i} \in 1 . . \mathrm{m}_{\mathrm{c}}} \mathrm{x}_{\mathrm{i}}^{\mathrm{c}}, \mathrm{z}=\Sigma_{\mathrm{j} \in 1 . \mathrm{n}_{\mathrm{c}}} \mathrm{Z}_{\mathrm{j}}^{\mathrm{c}}$ and $\mathrm{w}=\Sigma_{\mathrm{k} \in 1 . . \mathrm{c}} \Sigma_{\mathrm{i} \in 1 . . \mathrm{m}_{\mathrm{k}}} \Sigma_{\mathrm{j} \in 1 . . \mathrm{n}_{\mathrm{k}}}\left[\mathrm{x}_{\mathrm{i}}^{\mathrm{k}} \mathrm{Z}_{\mathrm{j}}^{\mathrm{k}}\left(\alpha_{\mathrm{k}}-\alpha_{\mathrm{k}-1}\right)\right]$. It can be shown that small changes in the longitudinal sections do not affect $\mathrm{F}_{r}\left(\theta, \mathrm{A}_{\theta}(\mathrm{v}), \mathrm{B}_{\theta}(\mathrm{v})\right)$ significantly (Matsakis and Nikitenko, to appear). Continuity is satisfied and, hence, robustness is achieved. Moreover, $\sum_{r \in \mathcal{A}} \mathrm{F}_{r}^{\mathrm{AB}}(\theta)$ measures to what extent the objects are involved in some spatial relationships along direction $\theta$. If this information is judged unimportant, the $\mathrm{F}_{r}$-histograms, of course, can be normalized. Let us denote by $\left\lceil\mathrm{F}_{r}^{\mathrm{AB}}\right\rfloor$ the histogram $\mathrm{F}_{r}^{\mathrm{AB}}$ after normalization ${ }^{3}$ :

$$
\forall \theta \in \mathbb{R},\left\lceil\mathrm{F}_{r}^{\mathrm{AB}}\right\rfloor(\theta)=\mathrm{F}_{r}^{\mathrm{AB}}(\theta) / \Sigma_{\rho \in \mathcal{A}} \mathrm{F}_{\rho}^{\mathrm{AB}}(\theta) .
$$

For a given direction $\theta$, the normalized $\mathrm{F}_{r}$-histograms define a fuzzy 13partition of the set of all object pairs, and each class of the partition corresponds to an Allen relation.

3 In Eqs. 1 and 2, we agree that a fraction is 0 if its denominator is 0. 


\section{Experiments}

In Figs. 6 and 7, a grayscale value is associated with each Allen relation (Fig. 6(a)). The thirteen normalized $\mathrm{F}_{r}$-histograms that represent the extracted directional and topological relationship information are plotted in the same diagram (Fig. 6(b)). The topological relationships along direction $\theta$ (on the $\mathrm{X}$-axis) are described by the vector composed of the thirteen $\left\lceil\mathrm{F}_{r}^{A B}\right\rfloor(\theta)$ values (on the Y-axis). Usually, most of these values are zero. The histograms are arranged in "layers." Several synthetic examples and histogram properties are presented in (Matsakis and Nikitenko, to appear).

Figure 7 represents a sequence of National Weather Service Detroit / Pontiac Doppler radar images. The sequence, captured on June 26, 2001, shows a mayfly aerial courtship over St. Clair County, Michigan (http://www.crh.noaa.gov/dtx/mayfly.htm). The argument is the mayfly swarm (light gray) and the referent is St. Clair County (dark gray). In (a), only the relations before and after are present; the swarm is born. In (b), the swarm becomes an unconnected object. The fragments close to and at the county border are responsible for the introduction of the relations $s$, $f, m, m i, o, o i$, and $d$. In (c), the swarm has grown considerably and moved over the county. The relation during clearly dominates at $\theta \approx 90^{\circ}$ and $\theta \approx$ $270^{\circ}$ with just a tiny bit of before and after caused by the single disjoint fragment below the county border. In $(d)$, the relation equals becomes more prominent for the near horizontal directions $\theta \approx 0^{\circ}$ and $\theta \approx 180^{\circ}$. Figure 7(e) shows that the only prominent relations are equals, during, starts, and finishes, which indicate that the swarm object is strictly contained by or is inner adjacent to the referent. Trace amounts of overlaps and overlapped by are still present, as the swarm object "spills over" the county boundary in some places. Note how before and after gradually diminishes as we progress from $(a)$ to $(e)$. Figure $7(f)$ demonstrates that $\mathrm{F}_{r}$-histograms can handle highly irregular and unconnected objects.

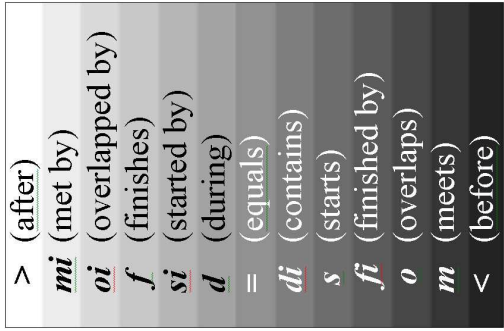

(a)

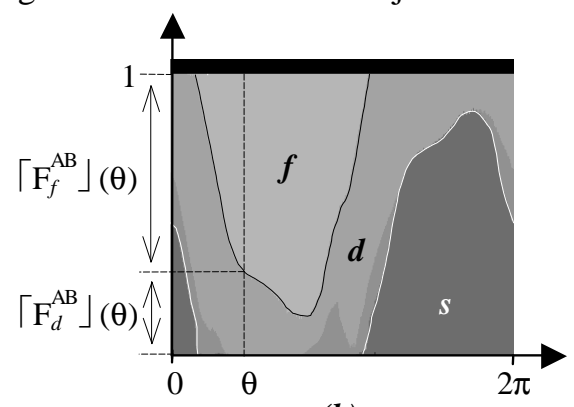

(b)

Fig. 6. (a) Allen relations and attached grayscale values. (b) An example of normalized $\mathrm{F}_{r}$-histogram. 


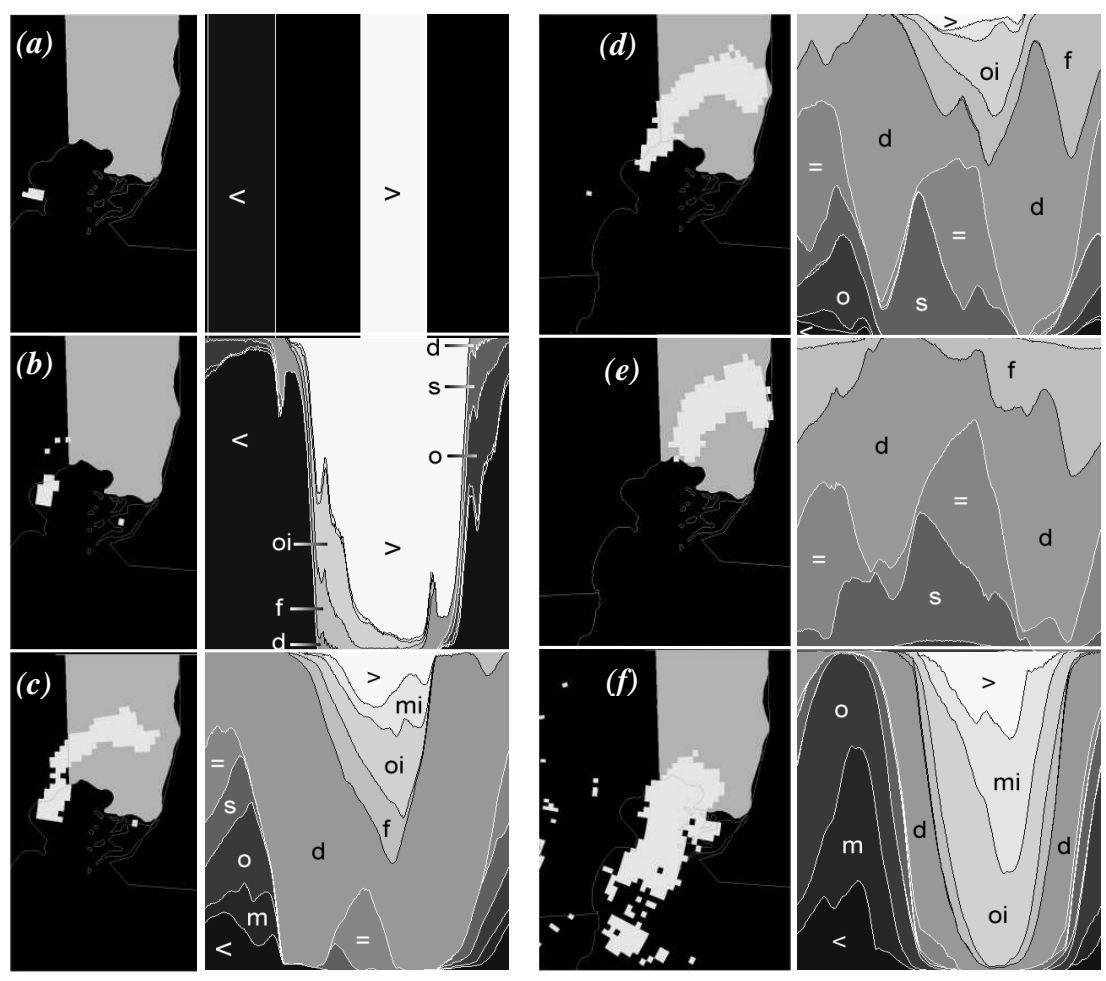

Fig. 7. Mayfly mating sequence captured by Doppler radar and the corresponding normalized $\mathrm{F}_{r}$-histograms.

\section{Towards a Linguistic Description of the Topological Relationships}

$\mathrm{F}_{r}$-histograms carry lots of topological relationship information. In (Matsakis et al. 2001), we used force histograms to generate a linguistic description of the relative position between two objects in terms of the four primitive directional relationships ("to the right of," "above," "to the left of," "below"). In future work, we plan to generate a linguistic expression that describes the topological relationships between two objects in terms of the thirteen Allen relations. Consider the direction $\theta=0^{\circ}$ and the object pair of Fig. 7(d). Is the argument before or contained by (during) the referent? Does it start, finish, or overlap it? All of these relations are present to some degree, but which one(s) give(s) the best description of the topological relationships between the two objects? The generated linguistic description 
should be terse and, at the same time, capture the essence of the relationships. Ultimately, it will consist of (i) a topological component to depict the relationships in terms of the most prominent Allen relations, (ii) a directional component to provide, if relevant, the direction where these relations hold true, (iii) a self-assessment component to give an indication of how satisfactory the description is-or how ambiguous the configuration is. None of these components are independent of each other. In this section, we focus our discussion on computing a satisfactory index for an arbitrary direction based on the Allen relations present along that direction.

\subsection{Coherent Sets of Allen Relations and Satisfactory Indices}

A linguistic expression like "A is before $\mathrm{B}$ in direction $\theta$ " might not describe satisfactorily a given configuration (another Allen relation might capture better the essence of the topological relationships between the two objects; the configuration might be ambiguous), but it certainly sounds coherent (it is easy to picture two such objects A and B). Although more complex, "A is mostly before but partially meets and overlaps B in direction $\theta$ " might also sound coherent to the reader. Three Allen relations are involved in this expression, but they do not semantically contradict each other. "A contains and is after B in direction $\theta$ " might seem less coherent. Whether a description sounds coherent or not is, of course, a subjective matter. Let us now formalize this discussion.

\subsubsection{Coherent Sets of Allen Relations}

We will say that a set of Allen relations is coherent iff it belongs to some subset $\mathrm{C}$ of the power set $2^{\mathcal{A}}$. The relations within a coherent set are considered not to semantically contradict each other and, therefore, might be used together in a linguistic description. Here are 3 possible choices for $\mathrm{C}$ :

$$
\begin{aligned}
& \mathrm{C}_{1}=\cup_{r \in \mathcal{A}}\{\{r\}\} \\
& \mathrm{C}_{2}=\mathrm{C}_{1} \cup\left\{\left\{r, r^{\prime}\right\} \subset \mathcal{A} \mid \delta_{r r^{\prime}}=1\right\} \\
& \mathrm{C}_{3}=\mathrm{C}_{2} \cup\left\{\left\{r, r^{\prime}, r^{\prime \prime}\right\} \subset \mathcal{A} \mid \delta_{r r^{\prime}}=\delta_{r r^{\prime \prime}}=\delta_{r^{\prime} r^{\prime \prime}}=1\right\}
\end{aligned}
$$

In these formulas, $\delta_{r r^{\prime}}$ denotes the conceptual distance between $r$ and $r^{\prime}$. It is the length of the shortest path between $r$ and $r^{\prime}$ in the graph of Fig. 3. For instance, $\boldsymbol{\delta}_{m m}=0, \delta_{m o}=1$ and $\boldsymbol{\delta}_{m f}=3$. The set $\mathrm{C}_{1}$ contains 13 singletons: $\{<\}$, $\{m\},\{o\}$, etc. By choosing $\mathrm{C}=\mathrm{C}_{1}$, we indicate that, in our opinion, coherent descriptions cannot involve more than one Allen relation. Therefore, an expression like "A is mostly before but partially meets $\mathrm{B}$ in direction $\theta$ " should not be produced by the system for linguistic description generation. The set $\mathrm{C}_{2}$ includes $\mathrm{C}_{1}$ and contains all pairs of neighbor relations (like $\{<, m\}$, but not $\{d i,>\}$ ). In the case where $\mathrm{C}=\mathrm{C}_{2}$, 
the above-mentioned expression might be generated. "A contains and is after B in direction $\theta$," on the other hand, will be rejected by the system. $\mathrm{C}_{3}$ also contains elements like $\{s, d, e q\}$ (but not $\{s, d, f\}$ ). There are, of course, other possible choices for $\mathrm{C}$. It seems reasonable to state that $\mathrm{C}$ should not contain the empty set and should include $\mathrm{C}_{1}$.

\subsubsection{Satisfactory Indices}

Several linguistic expressions can be associated with the same coherent set of Allen relations. For instance, "A is before $\mathrm{B}$ in direction $\theta$ " and "A is mostly before B in direction $\theta$ " are both associated with $\{<\}$ (we will not discuss here the problem of finding the most appropriate expression). Again, such descriptions might be coherent, but not satisfactory for the configuration in hand. For any relation $r$, let $\mathrm{v}_{r}$ denote the value $\left\lceil\mathrm{F}_{r}^{\mathrm{AB}}\right\rfloor(\theta)$. Here is the simplest way to attach a satisfactory index $\sigma_{\{r\}}$ to the coherent set $\{r\}$ (i.e., to the most appropriate description associated with $\{r\}$ ): $\sigma_{\{r\}}=\mathrm{v}_{r}$. One might argue, however, that $\sigma_{\{<\}}$should be higher when $\mathrm{v}_{<}=0.7$ and $\mathrm{v}_{m}=0.3$ (before and meets coexist) than when $\mathrm{v}_{<}=0.7$ and $\mathrm{v}_{>}=0.3$ (before and after coexist). $\sigma_{\{r\}}=\max \left(0, \mathrm{v}_{r}-\Sigma_{\rho \in \mathcal{A}-\{r\}}\left(\delta_{\rho r} / 6\right) \mathrm{v}_{\rho}\right)$ is another way to define a satisfactory index. Note that 6 is the maximum possible conceptual distance between two Allen relations. Any relation $\rho$ that coexists with $r$ makes $\sigma_{\{r\}}$ decrease, and the higher its distance to $r$, the bigger the decrease. $\sigma_{\{r\}}$ belongs to the interval $[0,1]$. It is 1 if and only if $\mathrm{v}_{r}$ is 1 ( $r$ is the only relation present), and cannot be 0 if $\mathrm{v}_{r}$ is greater than 0.5 . Generalization is easy, and a satisfactory index $\sigma_{\mathrm{c}}$ can be attached to any coherent set $\mathrm{c}$ of Allen relations. Here are two possible definitions:

$$
\begin{gathered}
\sigma_{\mathrm{c}}=\Sigma_{r \in \mathrm{c}} \mathrm{v}_{r}, \\
\sigma_{\mathrm{c}}=\max \left(0, \sum_{r \in \mathrm{c}} \mathrm{v}_{r}-\Sigma_{\rho \in \mathcal{A}-\mathrm{c}}\left(\delta_{\rho \mathrm{c}} / 6\right) \mathrm{v}_{\rho}\right)
\end{gathered}
$$

In Eq. $7, \delta_{\rho c}$ denotes a weighted average conceptual distance between the Allen relation $\rho$ and the coherent set c: $\delta_{\rho c}=\left(\Sigma_{r \in \mathrm{c}} \mathrm{v}_{r} \delta_{\rho r}\right) / \Sigma_{r \in \mathrm{c}} \mathrm{v}_{r}$. The index $\sigma_{\mathrm{c}}$ is a continuous function of all the $\mathrm{v}_{r}$ values. Moreover, if $\mathrm{c}=\mathrm{c}^{\prime} \cup\left\{r_{0}\right\}$ with $\mathrm{c}^{\prime}$ another coherent set and $r_{0}$ such that $\mathrm{v}_{r_{0}}=0$, then $\sigma_{\mathrm{c}}=\sigma_{\mathrm{c}^{\prime}}$. The transition between different coherent sets is also continuous.

\subsection{Examples and Future Work}

Consider the object pair in Fig. $7(d)$ and the direction $\theta=0^{\circ}$. Table 1 shows the highest satisfactory index for two different definitions of $\sigma_{\mathrm{c}}$ (Eqs. 6 and 7) and three different choices of $\mathrm{C}$ (Eqs. 3 to 5). Here, both definitions agree. In direction $\theta=0^{\circ}$, if the topological relationships between the swarm and the county had to be described by exactly one Allen relation, that relation should be equals. The description, however, 
TABLE 1.

Highest satisfactory index for the object pair in Fig. $7(d)$ and the direction $\theta=0^{\circ}$.

\begin{tabular}{|c|c|c|}
\hline \multicolumn{3}{|c|}{$\sigma_{\mathrm{c}}$ defined by Eq. 6} \\
\hline $\mathrm{C}$ & $\max _{\mathrm{c} \in \mathrm{C}} \sigma_{\mathrm{c}}$ & $\operatorname{argmax}_{\mathrm{c} \in \mathrm{C}} \sigma_{\mathrm{c}}$ \\
\hline $\mathrm{C}_{1}$ & 0.373 & $\{e q\}$ \\
$\mathrm{C}_{2}$ & 0.541 & $\{e q, f\}$ \\
$\mathrm{C}_{3}$ & 0.687 & $\{e q, f, d\}$ \\
\hline
\end{tabular}

\begin{tabular}{|c|c|c|}
\hline \multicolumn{3}{|c|}{$\sigma_{\mathrm{c}}$ defined by Eq. 7} \\
\hline $\mathrm{C}$ & $\max _{\mathrm{c} \in \mathrm{C}} \sigma_{\mathrm{c}}$ & $\operatorname{argmax}_{\mathrm{c} \in \mathrm{C}} \sigma_{\mathrm{c}}$ \\
\hline $\mathrm{C}_{1}$ & 0.247 & $\{e q\}$ \\
$\mathrm{C}_{2}$ & 0.426 & $\{e q, f\}$ \\
$\mathrm{C}_{3}$ & 0.595 & $\{e q, f, d\}$ \\
\hline
\end{tabular}

would not be very satisfactory. A better description would be obtained if equals, finishes and during were considered not to semantically contradict each other. As expected, Eq. 7 gives lower values than Eq. 6 due to the negative influence of the relations outside of the winning coherent sets.

Much work remains to be done before we can generate a linguistic description that captures the essence of the topological relationships between two complex objects in terms of the thirteen Allen relations. First, we intend to find a direction where a description would be most representative. Intuitively, the direction $\theta_{0}$ we seek maximizes both the highest satisfactory index $\max _{\mathrm{c} \in \mathrm{C}} \sigma_{\mathrm{c}}(\theta)$ and the degree $\Sigma_{r \in \mathcal{A}} \mathrm{F}_{r}^{\mathrm{AB}}(\theta)$ of object interaction. The linguistic expression generated by the system will involve the Allen relations comprising $\mathrm{c}_{0}$, the coherent set that maximizes $\sigma_{\mathrm{c}}\left(\theta_{0}\right)$. A fuzzy rule base will be used to produce the most appropriate expression given the values $\left\lceil\mathrm{F}_{r}^{\mathrm{AB}}\right\rfloor\left(\theta_{0}\right)$. Finally, the self-assessment component of the description will be derived from the satisfactory index $\sigma_{\mathrm{c}_{0}}\left(\theta_{0}\right)$.

\section{Conclusions}

The F-histogram is a powerful generic quantitative representation of the relative position between two $2 \mathrm{D}$ objects. In this paper, we have considered $\mathrm{F}_{r}$-histograms, which are dedicated to the extraction of directional and topological relationship information. Imprecision is handled and robustness achieved through fuzzy set theoretic approaches. For any direction in the plane, the $\mathrm{F}_{r}$-histograms define a fuzzy 13partition of all object pairs, and each class of the partition corresponds to an Allen relation. The objects are not necessarily convex, nor connected, and their geometry is not approximated through, e.g., Minimum Bounding Rectangles. Experiments on real data have shown that $\mathrm{F}_{r}$-histograms carry lots of topological relationship information. An index to measure the complexity of the relationships in an arbitrary direction has been developed. This index will play an important role in the generation of linguistic descriptions that capture the essence of the topological relationships between regions in terms of the Allen relations. 


\section{Acknowledgments}

The authors want to express their gratitude for support from the Natural Science and Engineering Research Council of Canada (NSERC), grant 045638 .

\section{References}

Allen JF (1983) Maintaining Knowledge about Temporal Intervals. Communications of the ACM 26(11):832-843

Dutta S (1991) Approximate Spatial Reasoning: Integrating Qualitative and Quantitative Constraints. International Journal of Approximate Reasoning 5:307-331

Freeman J (1975) The Modeling of Spatial Relations. Computer Graphics and Image Processing 4:156-171

Goodchild M, Gopal S (1990) (Eds.) The Accuracy of Spatial Databases. Taylor and Francis, Basingstoke, UK

Guesgen HW (2002) Fuzzifying Spatial Relations. In: Matsakis P, Sztandera L (Eds.) Applying Soft Computing in Defining Spatial Relations. Studies in Fuzziness and Soft Computing, Physica-Verlag, 106:1-16

Malki J, Zahzah EH, Mascarilla L (2002) Indexation et recherche d'image fondées sur les relations spatiales entre objets. Traitement du Signal 18(4)

Matsakis P (1998) Relations spatiales structurelles et interprétation d'images. $\mathrm{Ph}$. D. Thesis, Institut de Recherche en Informatique de Toulouse, France

Matsakis P (2002) Understanding the Spatial Organization of Image Regions by Means of Force Histograms: A Guided Tour. In: Matsakis P, Sztandera L (Eds.) Applying Soft Computing in Defining Spatial Relations. Studies in Fuzziness and Soft Computing, Physica-Verlag, 106:99-122

Matsakis P, Keller J, Wendling L, Marjamaa J, Sjahputera O (2001) Linguistic Description of Relative Positions in Images. IEEE Transactions on Systems, Man and Cybernetics, Part B 31(4):573-588

Matsakis P, Nikitenko D (to appear) Combined Extraction of Directional and Topological Relationship Information from 2D Concave Objects. In: Cobb M, Petry F, Robinson V (Eds.) Fuzzy Modeling with Spatial Information for Geographic Problems, Springer-Verlag Publications

Matsakis P, Wendling L (1999) A New Way to Represent the Relative Position between Areal Objects. IEEE Transactions on Pattern Analysis and Machine Intelligence 21(7):634-643

Nabil M, Shepherd J, Ngu AHH (1995) 2D Projection Interval Relationships: A Symbolic Representation of Spatial Relationships. SSD '95 (Advances in Spatial Databases: 42nd Symposium) pp. 292-309

Sharma J, Flewelling FM (1995) Inferences from Combined Knowledge about Topology and Direction. SSD'95 (Advances in Spatial Databases: 42nd Symposium) pp. 279-291 